\title{
Observer agreement for the diagnosis of intestinal acute graft-vs.-host disease based on the presence of villous atrophy in the terminal ileum
}

\author{
YUUSAKU SUGIHARA $^{1,2}$, SAKIKO HIRAOKA ${ }^{1}$, ERIKO YASUTOMI ${ }^{1}$, SHOHEI OKA ${ }^{1}$, YASUSHI YAMASAKI ${ }^{1}$, \\ TOSHIHIRO INOKUCHI ${ }^{1}$, HIDEAKI KINUGASA ${ }^{3}$, MASAHIRO TAKAHARA ${ }^{1}$, YUKI MORITO ${ }^{4}$, \\ SAKUMA TAKAHASHI $^{5}$, KEITA HARADA ${ }^{1}$, TAKEHIRO TANAKA ${ }^{6}$, FUMIO OTSUKA $^{2}$ and HIROYUKI OKADA ${ }^{1}$ \\ Departments of ${ }^{1}$ Gastroenterology and Hepatology, and ${ }^{2}$ General Medicine; ${ }^{3}$ Division of Endoscopy, Okayama University \\ Graduate School of Medicine, Dentistry and Pharmaceutical Sciences, Okayama 700-8558; ${ }^{4}$ Department of \\ Gastroenterology and Hepatology, Hiroshima City Hiroshima Citizens Hospital, Hiroshima 730-8518; \\ ${ }^{5}$ Department of Gastroenterology and Hepatology, Kagawa Prefectural Central Hospital, Takamatsu, Kagawa 760-8557; \\ ${ }^{6}$ Department of Diagnostic Pathology, Okayama University Graduate School of Medicine, \\ Dentistry and Pharmaceutical Sciences, Okayama 700-8558, Japan
}

Received February 18, 2019; Accepted January 10, 2020

DOI: $10.3892 /$ etm.2020.8538

\begin{abstract}
Intestinal graft-vs.-host disease (GVHD) is a serious complication of allo-hematopoietic stem cell transplantation (allo-HSCT). Villous atrophy in the terminal ileum is considered a useful diagnostic indicator for GVHD. However, the inter- and intra-observer agreement regarding the ileocolonoscopic findings indicative of acute intestinal GVHD, i.e., villous atrophy in the terminal ileum, are currently insufficient in multiple institutions. Thus, the present study aimed to investigate the incidence of villous atrophy in the terminal ileum to diagnose acute intestinal GVHD and determine the inter- and intra-observer agreement regarding this result for experienced endoscopists from multiple institutions. Consecutive patients who underwent allo-HSCT were referred to our institution between May 2008 and September 2015. A total of 54 patients underwent total ileocolonoscopy after allo-HSCT due to suspected intestinal acute GVHD. Subsequently, three observers from different institutions evaluated the cases for the presence of villous atrophy in the terminal ileum. In this study, the pathology results were a gold standard to evaluate the predictive value of ileocolonoscopy
\end{abstract}

Correspondence to: Dr Yuusaku Sugihara, Department of Gastroenterology and Hepatology, Okayama University Graduate School of Medicine, Dentistry and Pharmaceutical Sciences, 2-5-1 Shikata-cho, Kita-ku, Okayama 700-8558, Japan

E-mail: y.sugi117@gmail.com

Abbreviations: GVHD, graft-vs.-host disease; allo-HSCT, allo-hematopoietic stem cell transplantation; PEG, polyethylene glycol

Key words: graft-vs.-host disease, terminal ileum, allo-hematopoietic stem cell transplantation, villous atrophy, endoscopy detection. Definitive pathological and non-pathological GVHD was diagnosed in 22 and 32 cases, respectively. The results of examining whether villous atrophy could predict GVHD were as follows. For three observers (A, B and C), the sensitivity of villous atrophy in the terminal ileum was $86.4,77.3$ and $79.2 \%$, respectively, whereas the specificity was $62.5,62.5$ and $86.7 \%$, respectively. The positive predictive value (PPV) and negative predictive value (NPV) of villous atrophy for GVHD were as follows: The PPV of appearance was 61.3, 58.6 and $82.6 \%$, respectively, whereas the NPV was 87.0,80.0 and 83.9\%, respectively. Kappa coefficients for the inter-observer reliability were $0.85,0.63$ and 0.63 for observers $\mathrm{A}$ and $\mathrm{B}, \mathrm{A}$ and $\mathrm{C}$, and $\mathrm{B}$ and $\mathrm{C}$, respectively. The intra-observer kappa coefficient was 0.88 for observer A, 0.73 for observer B and 0.75 for observer C. A substantial observer agreement was achieved for the analysis of villous atrophy in the terminal ileum and the agreement for the predictive histological diagnosis was also excellent. Based on the results of the present study, identification of villous atrophy in the terminal ileum was a clinically effective diagnostic parameter, even if different endoscopists were involved in the diagnosis at multiple institutions. The present study was registered as a trial with the University Hospital Medical Information Network (UMIN; registration no. UMIN000025390).

\section{Introduction}

Allo-hematopoietic stem cell transplantation (allo-HSCT) has become an essential part of the standard therapeutic management for several hematological diseases and malignancies, including acute or chronic leukemia. An estimated 10-40\% of patients who undergo allo-HSCT develop significant clinical acute graft-vs.-host disease (GVHD) (1). Gastrointestinal GVHD usually occurs $\geq 3$ weeks after allo-HSCT and is characterized by profuse diarrhea, anorexia, nausea, vomiting, abdominal pain and gastrointestinal bleeding $(2,3)$. 
Accurate diagnosis of intestinal GVHD is required, which is based on histological findings, including crypt abnormalities, e.g. degeneration, dilatation, abscesses and crypt loss, which accompany epithelial apoptosis in the ileum and colon (4). Ileocolonoscopy is commonly performed, as patients suspected to have acute GVHD complain of gastrointestinal symptoms. Pathological examination is indispensable for the confirmation of GVHD diagnosis; however, treatment may be rapidly initiated after the preliminary endoscopic diagnosis. Previous studies have reported that classic endoscopic features, including orange-peel appearance, spotty redness, small mucosa sloughing and diffuse mucosal defect, are useful in diagnosing acute GVHD (5). It has been previously reported that villous atrophy of the terminal ileum is most useful for the diagnosis of GVHD (6). However, these previous studies did not address details of the random sequence and were performed at a single institution. Thus, the present study aimed to investigate the incidence of villous atrophy in the terminal ileum and the inter- and intra-observer agreement for this finding among experienced endoscopists in multiple centers.

\section{Materials and methods}

Patients. The present retrospective study comprised 261 consecutive patients who underwent allo-HSCT and were referred to the Okayama University Graduate School of Medicine (Okayama, Japan) between May 2008 and September 2015. Among them, 62 patients underwent colonoscopy and had symptoms suggestive of gastrointestinal GVHD, including anorexia, nausea, vomiting, watery diarrhea and abdominal pain. All patients underwent biopsies in each of the following segments regardless of the presence or absence of abnormalities that may be indicative of intestinal GVHD: Terminal ileum, right hemi-colon, left hemi-colon and rectum. A total of 6 patients who were unable to undergo total ileocolonoscopy due to incomplete preparation or unbearable pain were excluded from the study. In addition, two patients with inadequate imaging information following ileocolonoscopy were excluded (Fig. 1). Demographic information, details on the hematological condition, symptoms and histological findings were retrospectively obtained from the patients medical records. All study participants provided informed consent. The study was approved by the local ethics review committee (approval no. 1610-013) and was registered at the University Hospital Medical Network Clinical Trials Registry (UMIN-CTR; registration no. UMIN000025390).

Endoscopic protocol. Experienced endoscopists performed the ileocolonoscopy in all patients using Olympus endoscopes (CF-H260I, CF-Q260I, PCF-Q260AI, CF-HQ-290I, CF-H290I or PCF-290I; Olympus Optical). Ileocolonoscopic images were evaluated based on the presence of villous atrophy observed in the terminal ileum (Figs. 2 and 3). Regarding the preparation for ileocolonoscopy, all patients were administered polyethylene glycol (PEG) if oral ingestion was possible.

A total of 3 experienced endoscopists ( $>10$ years as a gastroenterologist) from multiple institutions (Okayama University, Kagawa Prefectural Central Hospital, and Hiroshima Citizens Hospital) served as observers $(A, B, C)$ and examined the images for the presence of villous atrophy in the terminal ileum. They were blinded to any patient information, including pathological diagnosis of GVHD, during the determination of atrophy. The 3 endoscopists were blindly evaluated to check for inter- and intra-observer agreement.

Pathological examination. Biopsy specimens from all patients were subjected to routine HE staining for pathological examination. The pathological diagnosis of GVHD was based on the following criteria: i) The presence of apoptotic bodies in the epithelium of the ileum, colon or rectum; ii) crypt abnormalities of degeneration, dilatation, abscesses or loss; iii) all histological findings were determined by HE staining and CD8 immunostaining (Fig. 4). In the present study, GVHD was diagnosed when all of these three previously listed criteria were met. The gold standard of GVHD diagnosis is positive pathological detection. A patient was diagnosed with GVHD if the pathological findings of any ileocolonoscopic samples met these criteria.

Statistical analysis. All analyses were performed using JMP version 11.2 (StataCorp). Statistical comparisons were performed using the Student's t-test. $\mathrm{P}<0.05$ was considered to indicate statistical significance. Reproducibility coefficients were analyzed using the kappa agreement coefficient. Intraand inter-observer agreement values were classified as follows: Poor $(\leq 0.20)$, slight (0.21-0.40), moderate (0.41-0.60), substantial (0.61-0.80) and excellent (0.81-1.00). Diagnostic values, the diagnostic value of the ileocolonoscopy observation result compared with the pathological result as a gold standard, were compared among observers and for each observer in terms of sensitivity, specificity, positive predictive value (PPV) and negative predictive value (NPV) by a $2 \times 2$ contingency table using the Student's t-test.

\section{Results}

Patient demographics. The characteristics of the 54 patients are presented in Table I. Definitive pathological and non-pathological GVHDs were identified in 22 and 32 patients, respectively. The mean patient age was 46 years and the youngest patient was aged 10 years (range, 10-67) years. The most common diagnosis was acute myeloid leukemia.

The results of examining whether villous atrophy could predict GVHD in all 54 sets of images were as follows. The sensitivity (which examines whether villous atrophy could predict GVHD) was $86.4,77.3$ and $79.2 \%$, respectively, and the specificity was $62.5,62.5$ and $86.7 \%$, respectively, for the three observers (A, B and C). Furthermore, the PPV was 61.3, 58.6 and $82.6 \%$, respectively, whereas the NPV was $87.0,80.0$ and $83.9 \%$, respectively (Tables II-IV).

Inter-observer agreement. Table $\mathrm{V}$ displays the inter-observer reliability of each observer. Kappa coefficients among observers A and B, A and C and B and C were $0.85,0.63$ and 0.63 , respectively (Table $\mathrm{V}$ ).

Intra-observer agreement. When analyzing the villous atrophy in all patients, the intra-observer kappa coefficient was determined to be 0.88 (95\% CI 0.83-0.94) for observer A, 0.73 (95\% CI 0.65-0.81) for observer B and 0.75 (95\% CI 0.70-0.81) for observer C (Table IV). 


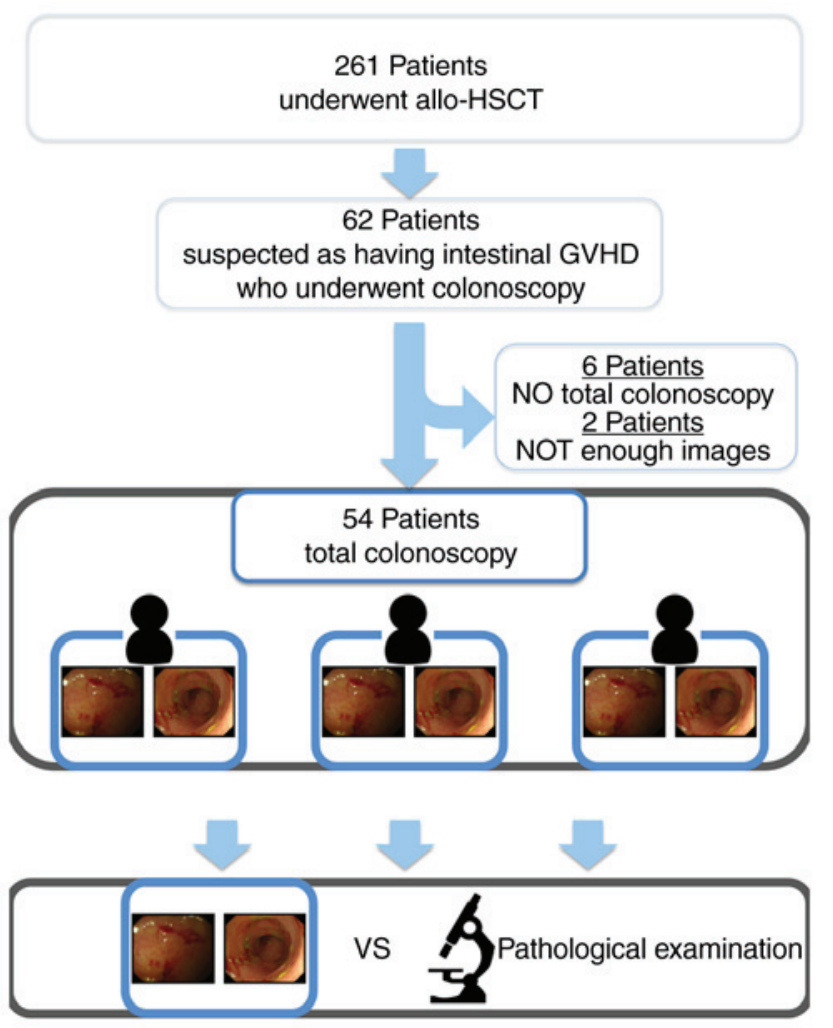

Figure 1. Flow diagram of the study. GVHD, graft-vs.-host disease; allo-HSCT, allo-hematopoietic stem cell transplantation.

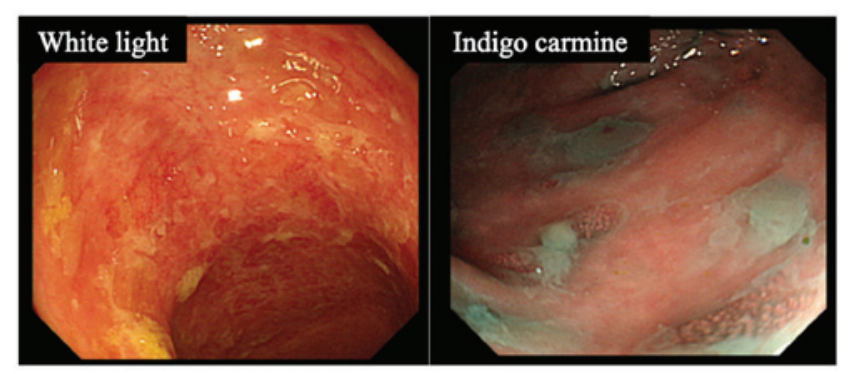

Figure 2. Endoscopic images with villous atrophy at the terminal ileum. Images are from the same patient pathologically diagnosed with GVHD. Villous atrophy was determined to be present when it was not possible to confirm villus contours at the terminal ileum using white light or indigo carmine images.

\section{Discussion}

The present study was the first, to the best of our knowledge, to investigate the inter- and intra-observer agreement for the ileocolonoscopic finding of villous atrophy in the terminal ileum to detect acute intestinal GVHD in multiple institutions.

A previous study by our group suggested that villous atrophy in the terminal ileum is a useful endoscopic finding in acute intestinal GVHD (6). Of note, the pathological diagnosis is indispensable for the final diagnosis of acute intestinal GVHD; however, the study by our group reported that villous atrophy in the terminal ileum is an important indicator for the estimation using ileocolonoscopy. Comparing this finding and the diagnosis of acute intestinal GVHD, the sensitivity of villous atrophy observed in the terminal ileum was $86.4,77.3$ and $79.2 \%$, whereas the specificity was $62.5,62.5$ and $86.7 \%$,

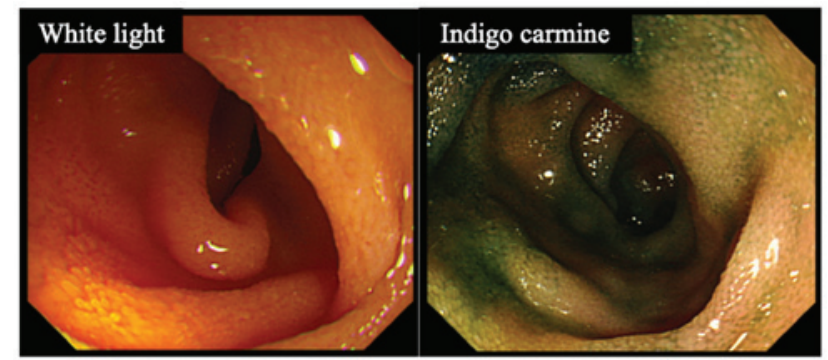

Figure 3. Endoscopic images without villus atrophy at the terminal ileum. Images are from the same patient who was not pathologically diagnosed with GVHD. Villous atrophy was determined to be absent when villous outlines were confirmed by white light or indigo carmine images at the end of the ileum.

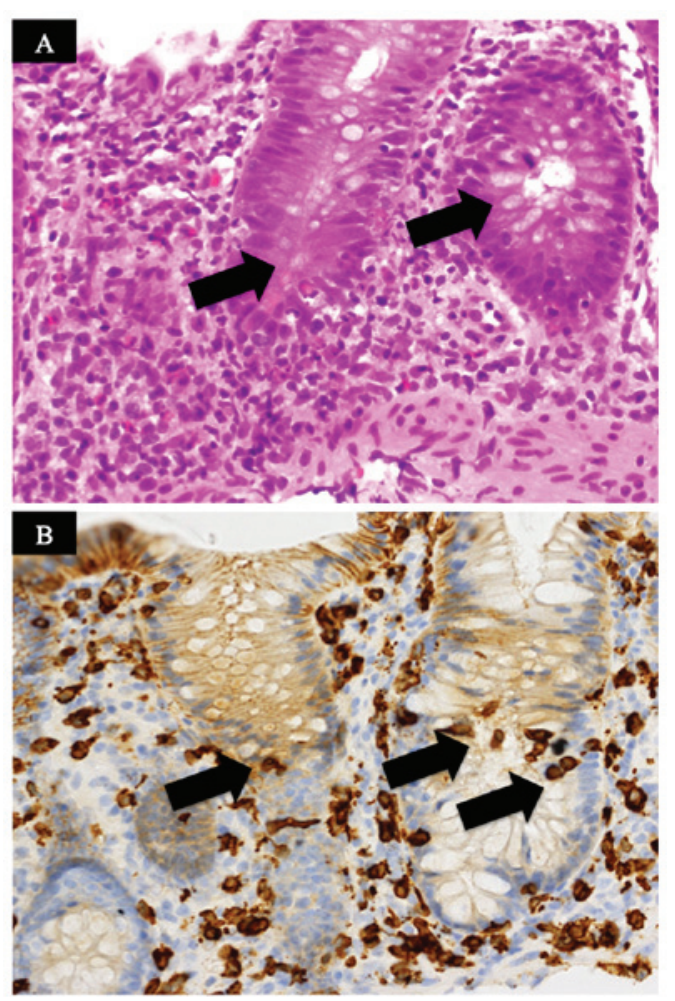

Figure 4. Pathological examination. (A) Histological observation of apoptotic bodies in the epithelial ileum (black arrows). (B) Cells positive for CD8 on immunostaining observed in the abnormal crypt with abscess formation (black arrows). Magnification, x400.

for observers A, B and C, respectively. In a previous study, Altun et al (7) reported that lower endoscopic findings had a sensitivity and specificity of 40 and $0 \%$, respectively. However, this study only examined a low number of patients in only one facility and did not emphasize the importance of terminal ileum observation. According to the previous study by our group, villous atrophy in the terminal ileum is an important finding (6); therefore, the terminal ileum should be visualized during colonoscopy.

To the best of our knowledge, the present study was the first to examine the inter-observer agreement of ileocolonoscopic findings for predicting GVHD. The kappa value for inter-observer agreement was 0.85 for observers A and B, 0.63 for $\mathrm{A}$ and $\mathrm{C}$, and 0.63 for $\mathrm{B}$ and $\mathrm{C}$. Based on these results, the inter-observer agreement was substantial to excellent in 
Table I. Characteristics of patients suspected to have gastrointestinal GVHD who underwent colonoscopy.

\begin{tabular}{lc}
\hline Item & Value \\
\hline Pathological result & \\
GVHD & 22 \\
No GVHD & 32 \\
Age (years), mean (range) & $46(10-67)$ \\
Sex & \\
Male & 32 \\
Female & 22
\end{tabular}

Disease requiring transplantation

$\begin{array}{ll}\text { Acute myeloid leukaemia } & 15\end{array}$

Myelodysplastic syndromes $\quad 11$

Acute lymphoblastic leukaemia $\quad 8$

Adult T-cell leukemia/lymphoma $\quad 4$

T lymphoblastic leukemia/lymphoma 4

B lymphoblastic leukemia/lymphoma 3

Chronic myelogenous leukaemia 3

Diffuse large B-cell lymphoma 1

Myelofibrosis

Non-Hodgkin lymphoma

Extranodal NK/T-cell lymphoma

Polycythaemia

Severe combined immunodeficiency

Stem cell source

Bone marrow

Cord blood

Peripheral blood stem cell

3

1

1

1

39

9

6

$\mathrm{n}=54$. Values are expressed as $\mathrm{n}$ unless otherwise specified. GVHD, graft-vs.-host disease; NK, natural killer.

Table II. Association between the number of patients with GVHD and villous atrophy by Observer A.

\begin{tabular}{lrrr}
\hline & \multicolumn{2}{c}{ GVHD } & \\
\cline { 2 - 3 } Villous atrophy & Positive & Negative & Total \\
\hline Positive & 19 & 12 & 31 \\
Negative & 3 & 20 & 23 \\
Total & 22 & 32 & 54
\end{tabular}

Sensitivity, $86.4 \%$; specificity, $62.5 \%$; positive predictive value, $61.3 \%$; negative predictive value, $87.2 \%$. GVHD, graft-vs.-host disease.

clinical practice. If an endoscopist has a certain amount of clinical experience, villous atrophy in the terminal ileum may be considered to have an excellent inter-observer agreement, even if the observers are from different institutions.

Certain endoscopists may argue that complete ileocolonoscopy, which requires insertion into the terminal ileum, is not
Table III. Association between the number of patients with GVHD and villous atrophy by Observer B.

\begin{tabular}{lrcc}
\hline & \multicolumn{2}{c}{ GVHD } & \\
\cline { 2 - 3 } Villous atrophy & Positive & Negative & Total \\
\hline Positive & 17 & 12 & 29 \\
Negative & 5 & 20 & 25 \\
Total & 22 & 32 & 54 \\
\hline
\end{tabular}

Sensitivity, $77.3 \%$; specificity, $62.5 \%$; positive predictive value, $58.6 \%$; negative predictive value, $80.0 \%$. GVHD, graft-vs.-host disease.

Table IV. Association between the number of patients with GVHD and villous atrophy by Observer C.

\begin{tabular}{lrcc}
\hline & \multicolumn{2}{c}{ GVHD } & \\
\cline { 2 - 3 } Villous atrophy & Positive & Negative & Total \\
\hline Positive & 19 & 4 & 23 \\
Negative & 5 & 26 & 31 \\
Total & 24 & 30 & 54 \\
\hline
\end{tabular}

Sensitivity, $79.2 \%$; specificity, $86.7 \%$; positive predictive value, $82.6 \%$; negative predictive value, $83.9 \%$. GVHD, graft-vs.-host disease.

Table V. Parameters of intraobserver and interobserver agreement regarding villous atrophy.

A, Inter-observer agreement (Kappa coefficient)

\begin{tabular}{llcc}
\hline Observer & A & B & C \\
\hline A & 1 & 0.85 & 0.63 \\
B & 1 & 0.63 & \\
C & 1 & & \\
\hline
\end{tabular}

$\mathrm{B}$, Intra-observer agreement

\begin{tabular}{lccc}
\hline Observer & $\mathrm{A}$ & $\mathrm{B}$ & $\mathrm{C}$ \\
\hline Kappa value & 0.88 & 0.73 & 0.75 \\
$95 \%$ CI & $0.83-0.94$ & $0.65-0.81$ & $0.70-0.81$ \\
\hline
\end{tabular}

widely accepted, as it is completely invasive and associated with a high risk of complications (8). However, we believe that careful endoscopic insertion techniques will enable rapid endoscopic diagnosis of acute intestinal GVHD. It may be speculated that pathological examination of acute intestinal GVHD is required for the diagnosis; however, endoscopic evaluation is also important, as the results may be obtained faster compared with pathological examination. In a previous study, endoscopic and histological findings of distal colonoscopy were indicated to be 
clinically significant in diagnosing patients who had intestinal GVHD with a poor general condition and who were at high risk of developing endoscopy-associated complications (9). Depending on the patient's condition, it is possible to prepare a testable condition by carefully prepping patients with PEG and enema. The method is to reduce the amount of food taken by patients and taking PEG on the day before the examination. On the day of examination, patients should take PEG carefully while paying attention to their symptoms. Prepping of patients is complete when the patient has taken $\sim 2,000 \mathrm{ml}$ of PEG and solid excretion has almost disappeared. Enema is performed if patients are not adequately prepped before examination

The present study had several limitations. First, since it was a retrospective study, images of the terminal ileum were already acquired. The location and angle of images were different for each endoscopist, which may have had an influence on the results of the present study. Furthermore, only 54 cases were screened by three observers, and it was not evaluated whether this number of patients and the number of observers were appropriate. Finally, the present study does not consider GVHD with cytomegalovirus or thrombotic microangiopathy. To the best of our knowledge, no studies have been performed on how the mucosa at the terminal ileum changes in GVHD with the above diseases. The study on the ileal mucosa of these diseases is an important theme for the future.

In conclusion, substantial inter- and intra-observer agreement was achieved for the detection of villous atrophy in the terminal ileum and the inter-observer agreement for the predictive histological diagnosis was also substantial to excellent, whereas the intra-observer agreement ranged from substantial to excellent for villous atrophy in the terminal ileum and acute intestinal GVHD. Based on the results of the present study, villous atrophy in the terminal ileum was a clinically effective diagnostic parameter even if different endoscopists from multiple institutions were involved in the diagnosis.

\section{Acknowledgements}

Not applicable.

\section{Funding}

No funding was received.

\section{Availability of data and materials}

The datasets used and/or analyzed during the present study available from the corresponding author on reasonable request.

\section{Authors' contributions}

YS, SH, FO, and HO made substantial contributions to conception and design. YS, EY, SO, YY, TI, HK, MT and TT were involved in the acquisition of data. YS, YM, ST and KH analysed and interpretated the data. YS and SH were involved in drafting the manuscript and revising it critically for important intellectual content. All authors approved the final manuscript and agreed to be accountable for all aspects of the work.

\section{Ethics approval and consent to participate}

The Ethics Review Committee of Okayama University Graduate School of Medicine granted ethical approval for the study (approval no. 1013) and was registered in the University Hospital Medical Information Network Clinical Trials Registry (reference no. UMIN000025390). All participants provided written informed consent.

\section{Patient consent for publication}

Not applicable.

\section{Competing interests}

The authors declare that they have no competing interests.

\section{References}

1. Iqbal N, Salzman D, Lazenby AJ and Wilcox CM: Diagnosis of gastrointestinal graft-versus-host disease. Am J Gastroenterol 95: 3034-3038, 2000.

2. Strasser SI and McDonald GB: Gastrointestinal and hepatic complications. Hematopoietic cell transplant. 4th ed. Oxford, UK: Wiley, Blackwell Publishing: 2009

3. Nevo S, Enger C, Swan V, Wojno KJ, Fuller AK, Altomonte V, Braine HG, Noga SJ and Vogelsang GB: Acute bleeding after allogeneic bone marrow transplantation: Association with graft versus host disease and effect on survival. Transplantation 67: 681-689, 1999.

4. Epstein RJ, McDonald GB, Sale GE, Shulman HM and Thomas ED: The diagnostic accuracy of the rectal biopsy in acute graft-versus-host disease: A prospective study of 13 patients. Gastroenterology 8: 764-771, 1980.

5. Endo K, Fujishima F, Kuroha M, Moroi R, Onodera M, Naito T, Kanazawa Y, Kimura T, Shiga H, Kakuta Y, et al: Effective and less invasive diagnostic strategy for gastrointestinal GVHD. Endosc Int Open 6: E281-E291, 2018.

6. Sugihara Y, Hiraoka S, Fujii N, Takashima S, Yamasaki Y, Inokuchi T, Takahara M, Kuwaki K, Harada K, Tanaka T and Okada $\mathrm{H}$ : Villous atrophy in the terminal ileum is a specific endoscopic finding correlated with histological evidence and poor prognosis in acute graft-versus-host disease after allo-hematopoietic stem cell transplantation. BMC Gastroenterol 18: 111, 2018.

7. Altun R, Gökmen A, Tek İ, Soydan E and Kurt Yüksel M: Endoscopic evaluation of acute intestinal graft-versus-host disease after allogeneic hematopoietic cell transplantation. Turk J Gastroenterol 27: 312-316, 2016.

8. Daniel F, Hassoun L, Husni M, Sharara A, Soweid A, Barada K, Haffar B, Massoud R, Shaib Y, Al-Hashash J, et al: Site specific diagnostic yield of endoscopic biopsies in gastrointestinal graft-versus-host disease: A tertiary care center experience. Curr Res Transl Med 67: 16-19, 2019.

9. Oomori S, Takagi S, Kikuchi T, Utsunomiya K, Yokoyama H, Negoro K, Tohmiya Y, Aihara H, Yamada M, Takahashi S, et al: Significance of colonoscopy in patients with intestinal graft-versus-host disease after hematopoietic stem cell transplantation. Endoscopy 37: 346-350, 2005.

(i) (-) This work is licensed under a Creative Commons Attribution-NonCommercial-NoDerivatives 4.0 International (CC BY-NC-ND 4.0) License. 